\title{
Thermal Desorption of Au from $W(001)$ Surface
}

\author{
R. Beaszczyszyn, J. Chrzanowski and P.J. Godowski* \\ Institute of Experimental Physics, University of Wrocław \\ pl. Maxa Borna 9, 50-204 Wrocław, Poland
}

(Received July 11, 2002; in final form October 16, 2002)

\begin{abstract}
Adsorption of $\mathrm{Au}$ on $\mathrm{W}(001)$ at $450 \mathrm{~K}$ up to multilayer structures was investigated. Temperature programmed desorption technique was used in determination of coverage dependent desorption energy (region up to one monolayer). Results were discussed in terms of competitive interactions of $\mathrm{Au}-\mathrm{Au}$ and $\mathrm{Au}-\mathrm{W}$ atoms. Simple procedure for prediction of faceting behavior on the interface, basing on the desorption data, was postulated. It was deduced that the $\mathrm{Au} / \mathrm{W}(001)$ interface should not show faceting tendency after thermal treatment.
\end{abstract}

PACS numbers: 68.43.Vx, 68.55.Ac, 68.60.Dv

\section{Introduction}

Investigation of metal adsorption and condensation on metal surfaces are of great importance from technological (application) and scientific (basic research) point of view. The $\mathrm{Au} / \mathrm{W}(001)$ interface, an $\mathrm{fcc} / \mathrm{bcc}$ type was investigated in the past $[1,2]$. It is known that on the (001) surface an adsorption layer has periodicity determined by the substrate. Gold grows in pseudomorphic layer growth manner, i.e. the first adlayer has the density of $1 \times 10^{19} \mathrm{at} / \mathrm{m}^{2}$ close to the substrate rather than to $\mathrm{Au}(001)$. The second Au layer forces the rearrangement of the distorted hexagonal first layer $(p(2 \times 2)$ low energy electron diffraction (LEED) pattern) to another distorted hexagonal packing $(p(3 \times 1))$ without change in the coverage. For $\mathrm{Au}$ the $\mathrm{fcc} / \mathrm{bcc}$ interfacial orientation relationship was established as (111)fcc \| (001)bcc and [110]fcc || [001]bcc. Finally, these layers are metastable and coalesce into three-dimensional crystals upon heating above $800 \mathrm{~K}$. Understanding

*corresponding author; e-mail: pjg@ifd.uni.wroc.pl 
of the stability and morphology of the Au/W interface helps in the other studies with the sandwich structures as well. For example, investigations of surface diffusion of water over gold film on tungsten tip by means of the field electron microscopy (FEM) technique could be performed $[3,4]$.

Temperature programmed desorption (TPD) measurements showed quite resolved desorption peaks for the first and second monolayer at the interval of $1400-1500 \mathrm{~K}$ and around $1240 \mathrm{~K}$, respectively. In addition, near first monolayer, the desorption peak had structure indicating two different interactions between $\mathrm{Au}$ and $\mathrm{W}$ atoms. Published values of the desorption energy $E_{\mathrm{d}}$, which is a good measure for the strength of bonding, and of the desorption frequency $\nu$ are rewritten in the form of the table (Table I). The $E_{\mathrm{d}}$ and $\nu$ parameters determined from FEM studies [5] are also included in Table I. The best fit for each desorption curve was obtained by varying both $E_{\mathrm{d}}$ and $\nu$ with coverage $\theta$ (the complete analysis of the family of desorption curves [6]). The coverage dependence of the desorption energy and the pre-exponential (frequency) factor are understood on the basis of lateral interactions between adsorbed atoms. For the $\mathrm{Au} / \mathrm{W}(001)$ case, $E_{\mathrm{d}}$ and $\nu$ showed the compensation effect, i.e. these values follow linear coverage depen-

TABLE I

Selected TPD results of the $\mathrm{Au} / \mathrm{W}$ - microcrystal (microcr.), i.e. tungsten tip as the substrate, the $\mathrm{Au} / \mathrm{W}$ (poly) and the $\mathrm{Au} / \mathrm{W}(001)$ interface published in [1] and [5]: desorption energy $E_{\mathrm{d}}$ and frequency $\nu . \mathrm{R}-$ Redhead's analysis with the standard value of $\nu$; RI - Redhead's analysis with the pre-assumed $\nu$ value; CA - complete analysis. The data of W(001) were analyzed using different methods; the results of CA were read from the published graph. The coverage is expressed in adsorbate monolayers, AML, i.e. 1 AML corresponds to the most dense gold overlayer on W(001).

\begin{tabular}{c|c|c|c|c}
\hline \hline Substrate & Description & $E_{\mathrm{d}}$ in $\mathrm{eV} / \mathrm{at}$ & $\nu$ in $1 / \mathrm{s}$ & Method \\
\hline $\mathrm{W}$ (microcr.) & $\theta<0.12 \mathrm{AML}$ & $3.3 \pm 0.2$ & $1 \times 10^{9}$ & $\mathrm{FEM}$ \\
$\mathrm{W}$ (microcr.) & $\theta>1 \mathrm{AML}$ & $3.8 \pm 0.2$ & $1 \times 10^{10}$ & $\mathrm{FEM}$ \\
$\mathrm{W}$ (poly) & & $3.6 \pm 0.5$ & $1 \times 10^{13}$ & $\mathrm{R}$ \\
$\mathrm{W}$ (poly) & I state & $4.06 \pm 0.32$ & $1 \times 10^{13}$ & $\mathrm{R}$ \\
W(poly) & II state & $3.92 \pm 0.32$ & $1 \times 10^{13}$ & $\mathrm{R}$ \\
W(poly) & & 4.54 & $8.2 \times 10^{13}$ & $\mathrm{RI}$ \\
$\mathrm{W}(001)$ & low coverage limit & 4.40 & $8.2 \times 10^{13}$ & $\mathrm{RI}$ \\
$\mathrm{W}(001)$ & $\approx 0.5 \mathrm{AML}$ & 4.13 & $1 \times 10^{13}$ & $\mathrm{R}$ \\
$\mathrm{W}(001)$ & $\approx 1.0 \mathrm{AML}$ & 3.90 & $1 \times 10^{13}$ & $\mathrm{R}$ \\
$\mathrm{W}(001)$ & $\approx 1.5 \mathrm{AML}$ & 3.46 & $1 \times 10^{13}$ & $\mathrm{R}$ \\
$\mathrm{W}(001)$ & $\approx 2.0 \mathrm{AML}$ & 3.36 & $1 \times 10^{13}$ & $\mathrm{R}$ \\
$\mathrm{W}(001)$ & $\approx 0.0 \mathrm{AML}$ & 5.80 & $1 \times 10^{18}$ & $\mathrm{CA}$ \\
$\mathrm{W}(001)$ & $\approx 0.9 \mathrm{AML}$ & 3.40 & $1 \times 10^{8}$ & $\mathrm{CA}$ \\
$\mathrm{W}(001)$ & $\approx 2.0 \mathrm{AML}$ & 5.00 & $1 \times 10^{20}$ & $\mathrm{CA}$
\end{tabular}


dence alongln $[\nu(\theta)]=b E_{\mathrm{d}}(\theta)+c$, with constants $b$ and $c$ (e.g. [7]). In other words, $E_{\mathrm{d}}$ and $\nu$ vary with coverage in the way to keep the product $\nu \exp \left(-E_{\mathrm{d}} / R T\right)$ approximately constant (e.g. [8]).

The aim of this work was to re-examine the $\mathrm{Au} / \mathrm{W}(001)$ system mainly by careful TPD measurements with minor support of Auger electron spectroscopy (AES) and LEED observations. For as sure obtaining a uniform gold adlayer, the deposition temperature of $450 \mathrm{~K}$ was chosen. The adsorption was extended to higher coverages, exceeding two adsorbate monolayers (AML's). In addition to usual TPD procedure with a single rate of heating for changing coverage, a varying heating rate for one coverage scheme (e.g. [9]) was made. A detailed sight into the adsorption system was obtained on the basis of consideration of subtle changes of desorption energy with coverage, especially in low coverage limit.

\section{Experimental}

The experiments were performed in the home-made multi-method ultrahigh vacuum (UHV) chamber pumped by an ion pump with cold cathode $(500 \mathrm{l} / \mathrm{s}$, Leybold-Heraeus) and a sublimation pump with possibility of liquid nitrogen $\left(\mathrm{LN}_{2}\right)$ cooling. The base pressure was better than $1 \times 10^{-10}$ Torr, during the experiments it was always less than $5 \times 10^{-10}$ Torr. The chamber was equipped with 4 -grid LEED/AES assembly, a quadrupole mass spectrometer (QMG 412, Balzers) and the $\mathrm{Au}$ atomic beam source of simple construction.

The W(001) crystal was attached to the manipulator using four spot-welded tungsten wires ( $\varnothing 0.4 \mathrm{~mm}$ ); such construction enables heating the sample up to $1900 \mathrm{~K}$ by passing the dc current (up to $25 \mathrm{~A}$ ) through the holder. Flashing the sample over $2700 \mathrm{~K}$ was performed by electron bombardment from additional tungsten filament $(\varnothing 0.1 \mathrm{~mm})$. Temperature of the sample was measured using a W-5\%Re/W-26\%Re (C-type) thermocouple spot-welded to the rear side of the specimen. Designed temperature programmer utilizes the proportional-integral-derivative (PID) feedback algorithm, written on PC, working with the power supply of the model SM 1540-D (Delta Elektronika). For the sample under study, heating rates, $\beta$ of the range of $0.1-20 \mathrm{~K} / \mathrm{s}$ with a linear heating schedule $(\beta=\mathrm{d} T / \mathrm{d} t)$ could be achieved. The sample surface was cleaned by alternate annealing in oxygen ambient $\left(1 \times 10^{-8}\right.$ Torr $)$ and flashing over $2700 \mathrm{~K}$ in UHV. The surface cleanliness was checked by AES and the main contaminant - carbon - had content lower than 0.01 monolayers.

$\mathrm{Au}$ was evaporated from tungsten filament heated by dc current passing through the source assembly. Evaporation on the crystal was performed in repeatable cycles (source current, time) and the distance between Au source and crystal in deposition position was approximately $10 \mathrm{~mm}$. Assuming the sticking coefficient equal to unity, the flux could be changed from 0.001 to $0.003 \mathrm{AML} / \mathrm{s}$. An aperture in the front of the mass spectrometer limited the flux accepted by it to the central area ( $2 \mathrm{~mm}$ of diameter) of the sample. 


\section{Results}

The $\mathrm{W}(001)$ surface was kept at $T=450 \mathrm{~K}$ during exposition at different doses to ensure sufficient mobility of $\mathrm{Au}$ adatoms to reach their equilibrium sites. Figures 1 and 2 show two series of TPD spectra after background subtraction and smoothing, corresponding to different initial adsorbate coverages (parameter of the curve). Those sets of spectra was measured with the heating rate of $\beta=9.14 \mathrm{~K} / \mathrm{s}$. Figure 1 represents the spectra for coverages ranging from 0 to around one adsorbate monolayer and Fig. 2 for higher ones. Initially single, well-defined desorption peak increases in intensity and its maximum shifts towards higher and next to

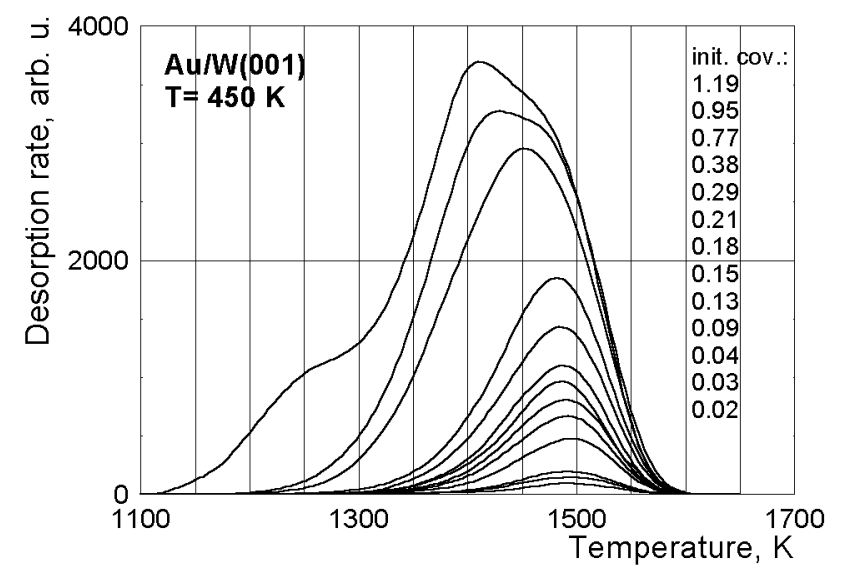

Fig. 1. Thermal desorption spectra with initial coverages (parameter of the curve) in adsorbate monolayers, AML. Heating rate was $9.14 \mathrm{~K} / \mathrm{s}$. See text for further details.

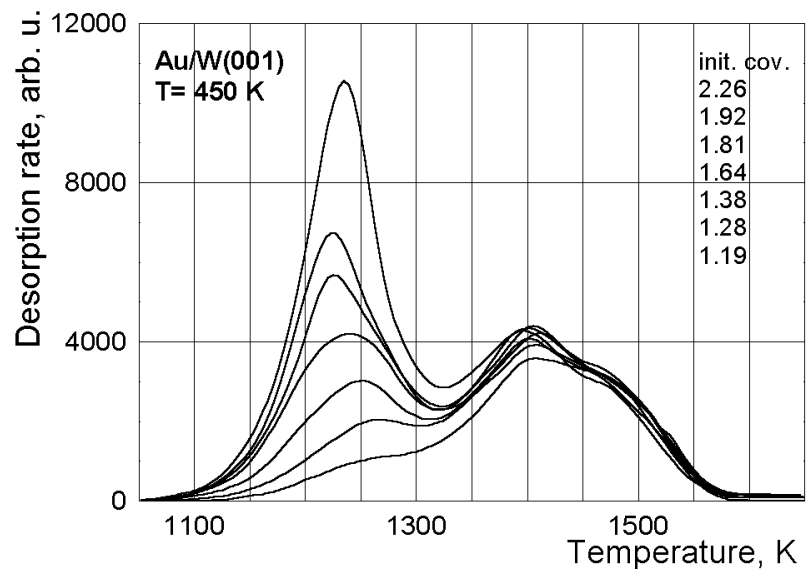

Fig. 2. Thermal desorption spectra with initial coverages (parameter of the curve) in adsorbate monolayers, AML. Heating rate was $9.14 \mathrm{~K} / \mathrm{s}$. See text for further details. 
lower temperature. Then, increasing in intensity peak shows double structure and the low temperature tail increases. For higher initial coverages, the second peak starts to grow up on the low temperature tail. Completion of the first Au monolayer corresponds to the curve which should be constructed between two highest ones of Fig. 1. The last spectrum of Fig. 1 has already a slight maximum indicative of the second adsorbate layer.

The calibration point was extracted from the dependence of the TPD peak height versus the area under the peak (not shown here). The peak height represents to some extent the degree of filling of the given adsorbate state and the area under the desorption spectrum corresponds to the number of desorbed (adsorbed) atoms. At the beginning, the height shows linear increase with the area. Then, two changes of slope could be seen and the crossing point of two last lines was attributed to the area (coverage) corresponding to a complete first layer. It could be seen from Figs. 1 and 2 that with increasing number of doses, the high temperature feature still slightly increases. It indicates that at the beginning the first Au monolayer has smaller density than it could have together with the second layer. It seems that with adsorption into second layer the first one rearranges into more dense layer. The area corresponding to the dense Au layer was established on the basis of the height-area graph as well. Surface coverage was then evaluated using the mentioned above criterion on the area under desorption peak. It is worthy mentioning that such procedures are commonly used in TPD (e.g. [10]). For coverages greater than one monolayer, the spectra show wide peak, which slightly sharpens with $\theta$ and finally its maximum shifts towards higher temperature. The top spectrum of Fig. 2 corresponds to the initial Au coverage evaluated as 2.26 AML. The spectra of higher gold coverages, not presented here, show that contributions of the individual layers $(\theta>2 \mathrm{AML})$ are not resolved. The multilayer desorption peak reaches its maximum temperature corresponding to the gold heat of sublimation.

A common method to determine the order of the desorption kinetics is to construct so-called "order plots" derived from a family of desorption spectra at different initial coverages [11-15]. The starting point of the derivation involves the application of the Polanyi-Wigner equation

$$
R=\nu(\theta) \theta^{n} \exp \left(-E_{\mathrm{d}} / k T\right),
$$

where $R$ is the desorption rate and $n$ is the order of the reaction of desorption. The slope for a plot of $\ln R$ as a function of $\ln \theta$ at constant temperature ("isotherms") reflects the order $n$. Figure 3 shows the order-plots constructed for TPD spectra of Fig. 1. For coverages lower than $0.4 \mathrm{AML}$, the slope of $1.05-1.21$ was determined. At higher coverages and lower temperatures the slope seems to increase and as it was determined approaches 1.5 value. Because of functional dependence of $E_{\mathrm{d}}(\theta)$ and $\nu(\theta)$, order plots are valuable for examining desorption phenomena at low coverages and higher temperatures [12]. On the basis of above results, for the $\mathrm{Au} / \mathrm{W}(001)$ interface there is no doubt that first-order process is involved. This 


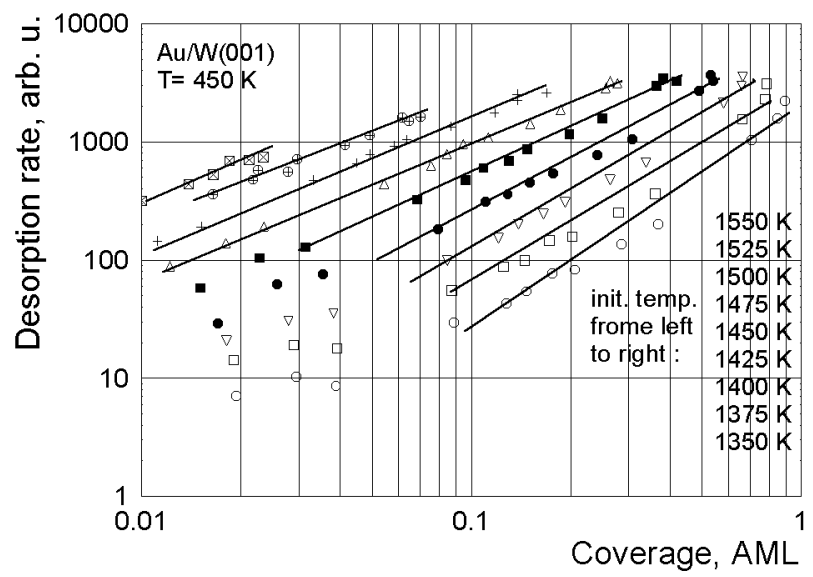

Fig. 3. Order plots for points of constant surface temperature constructed from the data presented in Fig. 1. The parameter is the desorption temperature.

statement is in agreement with the FEM study [5] which also provided the result $n=1$ for the Au submonolayer coverage range.

In order to evaluate coverage-dependent activation energies for desorption and frequency factors, the Bauer procedure was adopted [1]. As it is stated above, assumption for the use of the method requiring $n=1$ and independence of $\theta$ is ful-

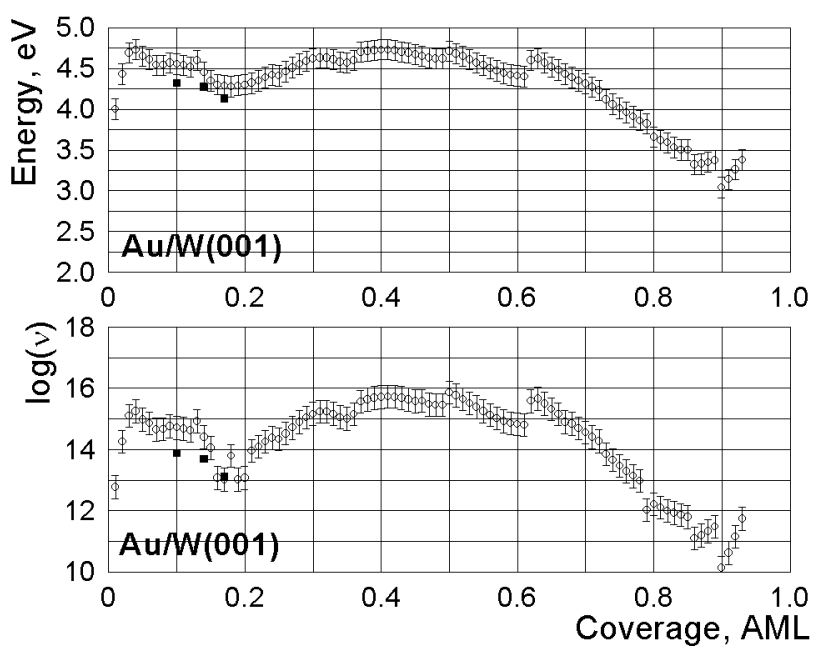

Fig. 4. Results of complete analysis of TPD spectra of Au adsorbed on W(001) at $T=450 \mathrm{~K}$. Upper panel represents activation energy of desorption and lower panel log of frequency factor as a function of gold coverage. The points of the spectra analysis of changing heating rate at constant initial coverage (squares) are included in the graphs. 
filled. For the family curves of Fig. 1 both dependencies $E_{\mathrm{d}}(\theta)$ and $\nu(\theta)$ are drawn in Fig. 4. For both functions we find a parallel behavior which indicates a compensation effect. At low coverages, the activation energy for desorption increases from 4.0 to $4.73 \mathrm{eV}$ and then after ca. $0.6 \mathrm{AML}$ decreases to $3.04 \mathrm{eV}$. Accordingly, the $\log$ of frequency factor changes from 12.8 to 15.3 and then decreases to 10.1.

To check the consistence of obtained data, three additional sets of desorption spectra were recorded with varying of the heating rate $\beta$ for a fixed initial surface coverage. Figure 5 shows the data for initial coverages of $0.10,0.14$, and 0.17 AML as calibrated by previous desorption experiment. The effect of changing sweeping rate is a variation of the peak temperature, e.g. for $0.10 \mathrm{AML} T_{\mathrm{p}}$ shifts by $43 \mathrm{~K}$ $(\approx 3 \%$ of peak temperature) with $\beta$ ranging from 4.60 to $12.9 \mathrm{~K} / \mathrm{s}$. Increasing the heating rate tends to increase the desorption rate, or equivalently the intensity as well [11], but the intensity-time area for any peak is consistent from experiment
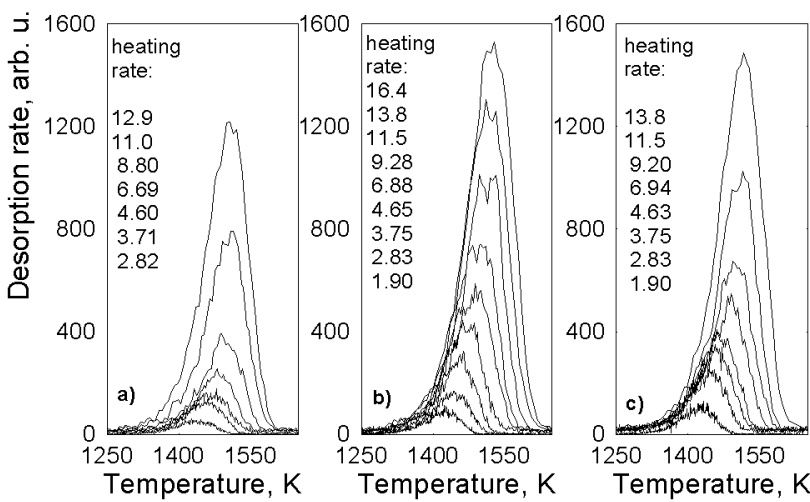

Fig. 5. Thermal desorption spectra of Au from W(001) obtained with a variable heating rate (parameter of curves), in $\mathrm{K} / \mathrm{s}$, for three different initial coverages: (a) $0.1 \mathrm{AML}$, (b) 0.14 AML and (c) 0.17 AML. The adsorption temperature is $450 \mathrm{~K}$.

TABLE II

The desorption parameters of the $\mathrm{Au} / \mathrm{W}(001)$ interface determined from experiment with the varying heating rate. The initial coverage is expressed in adsorbate monolayers (AML), the desorption energy $E_{\mathrm{d}}$ in $\mathrm{eV} /$ at and $\nu$ in $1 / \mathrm{s}$.

\begin{tabular}{c|c|c|c}
\hline \hline Initial coverage & $E_{\mathrm{d}}$ & $\nu \times 10^{13}$ & $\log \nu$ \\
\hline 0.10 & 4.32 & 7.63 & 13.88 \\
0.14 & 4.27 & 4.88 & 13.69 \\
0.17 & 4.13 & 1.25 & 13.1
\end{tabular}


to experiment. From the graph it may be seen that two lowest sweeping rates can give high uncertainty in $T_{\mathrm{p}}$ determination because of the decreased signal-to-noise ratio. For the remaining results, from the Arrhenius plot of $1 / T_{\mathrm{p}}$ versus $\ln \left(T_{\mathrm{p}}^{2} / \beta\right)$, the desorption energy $E_{\mathrm{d}}$ and the frequency factor $\nu$ are obtained [9] and the results are collected in Table II. The points are added to the plot in Fig. 4.

\section{Discussion}

TPD results can be discussed in terms of interaction of $\mathrm{Au}-\mathrm{W}$ and $\mathrm{Au}-\mathrm{Au}$ on the atomic level. The obtained spectra show well-defined desorption peaks for the first and second monolayer. At the beginning of adsorption (Fig. 4), desorption energy increases around $16 \%$ of its original value. It means that the strength of $\mathrm{Au}-\mathrm{W}$ interaction (dominating for $\theta \leq 0.05 \mathrm{AML}$ ) is influenced by lateral $\mathrm{Au}-\mathrm{Au}$ interaction (long range at this point) leading to this increase in desorption energy. Then, local variations of $E_{\mathrm{d}}(\theta)$ could be attributed to morphological changes in the first gold atomic layer. For coverages greater than approximately half a monolayer, monotonic decrease in $E_{\mathrm{d}}$ (within the experimental error) is a consequence of short-range $\mathrm{Au}-\mathrm{Au}$ interaction. This interplay between adsorbate-adsorbate and adsorbate-substrate interaction leads to reduction of $\mathrm{Au}-\mathrm{W}$ bond. Desorption spectra of the second layer show peak positioned at lower temperature, i.e. further lowering interaction of gold adatoms (of 2 nd layer) with the substrate. Despite of that, the multilayer desorption peak, $T_{\mathrm{p}}(m)$, moves (back) towards higher temperature. This increase in desorption energy is a consequence of the fact that $\mathrm{Au}$ atoms prefer to be bonded to other Au atoms than to tungsten ones. This is not the case of the $\theta<1$ AML region, where stronger influence of the substrate is observed (not bulk-like Au arrangement).

The order among the temperatures at the maximum desorption

$$
\operatorname{av}\left\{T_{\mathrm{p}}(0 \leq \theta \leq 1)\right\}>T_{\mathrm{p}}(m)>\operatorname{av}\left\{T_{\mathrm{p}}(1 \leq \theta \leq 2)\right\},
$$

where an average of $T_{\mathrm{p}}(\theta)$ over indicated interval is taken as the representative temperature, could be a measure for the interaction in the $\mathrm{Au} / \mathrm{W}(001)$ interface. Basing on the ratio

$$
\tau=\left\{T_{\mathrm{p}}^{\prime}(2)-T_{\mathrm{p}}(m)\right\} /\left\{T_{\mathrm{p}}^{\prime}(1)-T_{\mathrm{p}}(m)\right\},
$$

where sign "prime" denotes the representative temperature of the indicated coverage region, tendency of a given interface to faceting process can be predicted. Faceting is defined as massive reconstruction from a planar morphology to a microscopically faceted surface upon heating to $T \geq 700 \mathrm{~K}$ (e.g. [16]). It is worthy mentioning that the position of the desorption maxima of the second layer relative to that of the multilayer is decisive. Certainly, a positive sign of $\tau$, determined from the TPD spectra, predicts faceting for the interface under study. Probably for small negative values $(-2<\tau<0)$ faceting will be observed as well. For $\tau<-2$ 
TABLE III

The average desorption temperatures in $\mathrm{K}$ corresponding to the initial coverages of one, $T_{\mathrm{p}}^{\prime}(1)$, two, $T_{\mathrm{p}}^{\prime}(2)$, and multilayer, $T_{\mathrm{p}}(m)$ of selected interfaces. $T_{\mathrm{p}}(m)$ is determined from the heat of sublimation, $\Delta H_{\mathrm{s}}$, in $\mathrm{eV} / \mathrm{at}$ (Web Elements Periodic Table; http://www.shef.ac.uk/chemistry/web-elements/). Calculated $\tau$ ratio (see text for definition) is correlated with faceting tendency of the interface.

\begin{tabular}{c|c|c|c|c|c}
\hline \hline & $\mathrm{Au} / \mathrm{W}(001)^{a}$ & $\mathrm{Au} / \mathrm{W}(001)^{b}$ & $\mathrm{Au} / \mathrm{W}(011)^{a}$ & $\mathrm{Ag} / \mathrm{W}(001)^{a}$ & $\mathrm{Pd} / \mathrm{W}(001)^{c}$ \\
\hline$T_{\mathrm{p}}^{\prime}(1)$ & 1450 & 1460 & 1450 & 1150 & 1655 \\
$T_{\mathrm{p}}^{\prime}(2)$ & 1245 & 1235 & 1330 & 980 & 1357 \\
$\Delta H_{\mathrm{s}}$ & 3.79 & 3.79 & 3.79 & 2.95 & 3.92 \\
$T_{\mathrm{p}}(m)$ & 1405 & 1405 & 1405 & 1103 & 1450 \\
$\tau$ & -3.56 & -3.09 & -1.67 & -2.62 & -0.45 \\
faceting & no & no & yes & no & yes \\
\hline
\end{tabular}

${ }^{a}[1],{ }^{b}$ this work, ${ }^{c}[17]$

the interface should not undergo faceting due to weak relative adsorbate-substrate interaction.

The quantities needed for estimation of $\tau$ are collected in Table III for selected interfaces. It is known that faceting depends on the crystallographic orientation of the surface [16]. As an example, the Au/W system shows faceting on W(011) but no faceting on $\mathrm{W}(001)$. According to the Table III it can be seen that evidence of massive reconstruction is found for $\mathrm{Pd} / \mathrm{W}(001)$ but none for $\mathrm{Ag} / \mathrm{W}(001)$. Basing on the presented data correlation between $\tau$ and prediction of faceting is reasonable.

\section{Conclusions}

Following conclusions could be drawn from the reported investigations: (1) Adsorption of $\mathrm{Au}$ on $\mathrm{W}(001)$ at temperature greater than room temperature, i.e. at $450 \mathrm{~K}$, shows layer by layer growth mode with stable morphology of the interface. (2) Overlayer growth process could be qualitatively analyzed in terms of atomic interactions. (3) The version of the varying heating rate of the TPD gives approximately equivalent information concerning desorption energy and frequency factor to the one obtained from the complete analysis. (4) Basing on TPD results it is possible to predict that the multilayer $\mathrm{Au} / \mathrm{W}(001)$ interface will not undergo faceting upon heating.

\section{Acknowledgments}

This work was supported by the University of Wroctaw under Project No. $2016 /$ W/IFD/98. 


\section{References}

[1] E. Baner, F. Bonczek, H. Poppa, G. Todd, Surf. Sci. 53, 87 (1975).

[2] E. Bauer, H. Poppa, G. Todd, P.R. Davis, J. Appl. Phys. 48, 3773 (1977).

[3] R. Bryl, Vacuum 48, 333 (1997).

[4] R. Bryl, R. Błaszczyszyn, Vacuum 54, 103 (1999).

[5] A. Cetronio, J.P. Jones, Thin Solid Films 35, 113 (1976).

[6] D.A. King, Surf. Sci. 47, 384 (1975).

[7] J.W. Niemantsverdriet, K. Markert, K. Wandelt, Appl. Surf. Sci. 31, 211 (1988).

[8] J.B. Miller, H.R. Siddiqui, S.M. Gates, J.N. Russell Jr., J.T. Yates Jr., J.C. Tully, M.J. Cardillo, J. Chem. Phys. 87, 6725 (1987).

[9] D. Edwards Jr., J. Appl. Phys. 46, 1444 (1975).

[10] A. Pavlovska, H. Steffen, E. Bauer, Surf. Sci. 195, 207 (1988).

[11] J.L. Taylor, W.H. Weinberg, Surf. Sci. 78, 259 (1978).

[12] T.S. Wittrig, D.E. Ibbotson, W.H. Weinberg, Appl. Surf. Sci. 4, 234 (1980).

[13] K. Nagai, Surf. Sci. 176, 193 (1986).

[14] A.M. deJong, J.W. Niemantsverdriet, Surf. Sci. 233, 355 (1990).

[15] D. Schlatterbeck, M. Parschau, K. Christmann, Surf. Sci. 418, 240 (1998).

[16] T.E. Madey, J. Guan, C.-H. Nien, C.-Z. Dong, H.-S. Tao, R.A. Campbell, Surf. Rev. Lett. 3, 1315 (1996).

[17] S. Prigge, H. Roux, E. Bauer, Surf. Sci. 107, 101 (1981). 